\title{
AVALIAÇÃo DE CARVÃo ATIVADO DE BABAÇU PARA A ADSORÇÃO SELETIVA DE CORANTE REATIVO BF-5G
}

\author{
C. A. CIONEK ${ }^{1}$, D. S. CARVALHO ${ }^{1,2}$, BARROS, M.A.S.D ${ }^{3}$. e P. A. ARROYO ${ }^{3}$ \\ ${ }^{1}$ Universidade Estadual de Maringá, Departamento de Engenharia Têxtil \\ ${ }^{2}$ Universidade Tecnológica Federal do Paraná , Coordenação de Engenharia Têxtil \\ ${ }^{3}$ Universidade Estadual de Maringá, Departamento de Engenharia Química \\ E-mail para contato: carolcionek@hotmail.com
}

\begin{abstract}
RESUMO - Na indústria têxtil é utilizada grande quantidade de água, que necessita de tratamento para retornar aos rios ou ser reutilizada no processo. Como tratamento desse efluente tem-se utilizado com grande êxito o processo de adsorção. Assim, o objetivo do trabalho foi estudar a utilização de carvão ativado de babaçu para a remoção seletiva do corante reativo azul BF-5G, por processo de adsorção em batelada, a $20{ }^{\circ} \mathrm{C}, 30{ }^{\circ} \mathrm{C}$ e 40 ${ }^{\circ} \mathrm{C}$. O carvão possui ponto de carga zero de 7,4 , microporosidade, com contribuição de mesoporos, e presença de grupos superficiais hidroxila, aldeído e cetona. A quantidade máxima adsorvida foi de $76,21 \mathrm{mg} \cdot \mathrm{g}^{-1}$ e os modelos de Sips e Freundlich se ajustaram melhor aos dados das isotermas. Os valores das propriedades termodinâmicas $\left(\Delta \mathrm{H}^{\circ} \mathrm{e}\right.$ $\Delta \mathrm{G}^{\circ}$ ) mostraram que o processo é espontâneo e endotérmico e a temperatura favorece o processo de adsorção, indicando que a contribuição da adsorção química no processo não deve ser desprezada.
\end{abstract}

\section{INTRODUÇÃO}

A indústria têxtil tem um papel importante na economia mundial. Esse segmento industrial desempenha diversas funções até que o produto final chegue ao consumidor. Para que um artigo têxtil apresente características de enobrecimento, é necessário que este seja beneficiado. Dentro do processo de beneficiamento de tecidos, são vários os processos que são utilizados água, como lavagem ou pré-tratamento, alvejamento, tingimento, estamparia e algum tipo de acabamento especial, porém, as principais fontes de poluição de águas residuais de têxteis vêm dos processos de tingimento (KHENIFI et al., 2007).

Atualmente são utilizados diversos métodos de remoção de corantes e de outros compostos químicos existentes no efluente, chamados em seu conjunto, de processos físico-químicos ou biológicos. Segundo Brás et al. (2002), dentre os processos físico-químicos, encontram-se a oxidação química (empregando como agente oxidante o cloro, a água oxigenada ou o ozônio), coagulação, floculação, precipitação, ultra, micro e nanofiltração por membranas, além de troca iônica e adsorção (em carvão ativado, rejeitos industriais e biosorventes). 
Há que se destacar os processos de adsorção que utilizam carvões ativados obtidos a partir de rejeitos industriais, estes estão sendo bastante empregados devido à versatilidade, eficiência e baixos custos de obtenção. Assim, o objetivo do trabalho foi estudar a utilização de carvão ativado de babaçu para a remoção seletiva do corante reativo azul BF-5G, por processo de adsorção em batelada, a $20^{\circ} \mathrm{C}, 30^{\circ} \mathrm{C}$ e $40^{\circ} \mathrm{C}$.

\section{MATERIAIS E MÉTODOS}

O adsorvente utilizado foi o carvão ativado proveniente do Coco de Babaçu (Tobasa Bioindustrial de Babaçu S.A.) e como adsorvato utilizou-se efluente sintético preparado com o corante reativo azul BF-5G, que é produzido pela Texpal Indústria Química S/A e foi gentilmente cedido.

\subsection{Cinética de Adsorção}

A partir da cinética de adsorção é possível determinar o tempo necessário para que o processo entre em equilíbrio e ajustar modelos aos dados obtidos que auxiliam avaliar o possível mecanismo de adsorção. Os ensaios foram realizados com concentração inicial das soluções de $60 \mathrm{mg} / \mathrm{L}$ e a agitação de $80 \mathrm{rpm}$. Os erlenmeyers contendo $20 \mathrm{~mL}$ de solução de corante e $0,2 \mathrm{~g}$ de adsorvente foram colocados em um banho termostatizado (Banho Dubnoff - Nova Ética), por um período total de $24 \mathrm{~h}$. Cada frasco foi retirado do banho em intervalos de tempo prédeterminados e a amostra foi filtrada. A concentração de cada amostra foi determinada por meio da leitura da absorbância em espectrofotômetro, no comprimento de onda de $620 \mathrm{~cm}^{-1}$. Assim, determinou-se o tempo necessário para se atingir o equilíbrio entre a solução de corante e o carvão ativado em questão. Os testes foram realizados em triplicata.

A quantidade adsorvida no tempo $t, \mathrm{q}_{\mathrm{ta}}(\mathrm{mg} / \mathrm{g})$, foi calculada por:

$$
q_{t a}=\frac{\left(C_{0}-C_{t a}\right) V}{M}
$$

sendo que $C_{0}$ e $C_{t a}(\mathrm{mg} / \mathrm{L})$ são a concentração de corante na fase líquida inicial e no tempo $t$, respectivamente, $V$ é o volume da solução (L) e $M$ é a massa de adsorvente $(\mathrm{g})$.

Para representar a cinética de remoção dos corantes no carvão ativado e ajustar os dados experimentais foram utilizados os modelos Pseudo-primeira ordem, Pseudo-segunda ordem e Difusão intrapartícula. Os parâmetros dos modelos foram calculados pelo software MICROCAL ORIGIN 8.

\subsection{Isotermas de adsorção}

Para a obtenção das isotermas foram realizados experimentos em triplicata, nos quais $0,2 \mathrm{~g}$ de adsorvente foram colocados em erlenmeyers contendo $20 \mathrm{~mL}$ de soluções de corante em concentrações que variaram entre 160 a $5000 \mathrm{mg} / \mathrm{L}$. Os erlenmeyers foram colocados em banho 
termostatizado na velocidade de agitação de $80 \mathrm{rpm}, \mathrm{a} 20^{\circ} \mathrm{C}, 30^{\circ} \mathrm{C}$ e $40{ }^{\circ} \mathrm{C}$, até que o equilíbrio fosse atingido. A quantidade de corante removida pelo adsorvente no equilíbrio, $\mathrm{q}_{\mathrm{ea}}(\mathrm{mg} / \mathrm{g})$, foi calculada pela equação (1).

Para o ajuste dos dados experimentais de equilíbrio obtidos para avaliar a influência da temperatura foram utilizados as isotermas de Langmuir, equação (2), Freundlich, equação (3), Tóth, equação (4), Radke-Prausnitz, equação (5), Redlich e Peterson, equação (6), e Sips, equação (7). Os parâmetros dos modelos foram calculados pelo software ORIGIN 8.

\begin{tabular}{|c|c|c|c|}
\hline Langmuir & $q_{e q}=\frac{q_{\max } K C_{e q}}{1+K C_{e q}}$ & $\begin{array}{l}\text { Radke e } \\
\text { Praunsnitz }\end{array}$ & $q_{\theta q}=\frac{K C_{\theta q} q_{m a x x}}{\left(1+K C_{\theta q}\right)^{n}}$ \\
\hline Freundlich & $q_{e q}=K C_{e q}^{1 / n}$ & Redlich Peterson & $q_{e q}=\frac{K C_{e q} q_{m a x x}}{1+a C_{e q}^{n}}$ \\
\hline Tóth & $q_{e q}=\frac{q_{m a ́ x} C_{e q}}{\left(\frac{1}{k}+C_{e q}^{n}\right)^{1 / n}}$ & Sips & $q_{e q}=\frac{\left(K C_{e q}\right)^{n} q_{m a x}}{1+\left(K C_{e q}\right)^{n}}$ \\
\hline
\end{tabular}

\subsection{Estudo termodinâmico}

Os valores das propriedades termodinâmicas $\Delta \mathrm{H}^{\circ}, \Delta \mathrm{S}^{\circ}$ e $\Delta \mathrm{G}^{\circ}$ foram calculados a partir das isotermas de adsorção de corante $\mathrm{q}_{\mathrm{e}}$ em função de $\mathrm{C}_{\mathrm{e}}$, assim, a partir dos dados de $\mathrm{q}_{\mathrm{e}}$ e $\mathrm{C}_{\mathrm{e}}$ foi calculado o valor de $\mathrm{K}_{0}$, sendo esse parâmetro a constante de equilíbrio termodinâmico. Para isto, foi utilizado o método proposto por Raji e Anirudhan (1998), que consiste em construir um gráfico de $\ln \left(\mathrm{q}_{\mathrm{e}} / \mathrm{C}_{\mathrm{e}}\right)$ versus $\mathrm{q}_{\mathrm{e}}$, extrapolando para $\mathrm{q}_{\mathrm{e}}=0$. Com o valor de $\mathrm{K}_{0}$ foi calculado a variação da energia livre de Gibs por meio da Equação (2) e a entropia e entalpia pela Equação (3).

$$
\begin{aligned}
& \ln K_{D}=\frac{\Delta S^{o}}{R}-\frac{\Delta H^{o}}{R T} \\
& \Delta G^{o}=\Delta H^{o}-T \Delta S^{o}
\end{aligned}
$$

\section{RESULTADOS E DISCUSSÃO}

\subsection{Cinética de adsorção}


Os modelos foram ajustados aos dados cinéticos nos modelos de pseudo-primeira ordem, pseudo-segunda ordem e difusão intrapartícula. A Figura 1 apresenta as curvas cinéticas experimentais para cada carvão ativado e os modelos cinéticos ajustados.

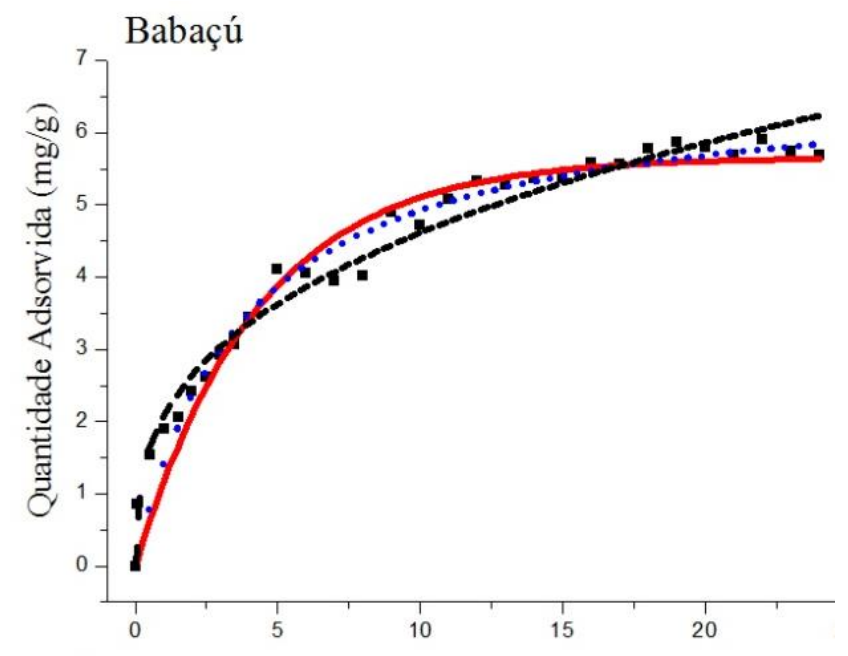

Figura 1 - Ajuste dos modelos de pseudo-primeira ordem, de pseudo-segunda ordem e difusão intrapartícula, para a remoção de corante.

Pelos resultados apresentados na Tabela 1 pode-se observar que o modelo cinético pseudoprimeira ordem não representou apropriadamente o comportamento da cinética de remoção, pois apresentou um valor do coeficiente de regressão baixo. Ao observar o coeficiente de correlação do modelo de difusão intrapartícula nota-se que este apresentou os melhores valores, porém, há de se levar em conta os valores negativos de $q_{e q}$, verificando que, assim como nos demais carvões, o modelo que melhor representou os dados experimentais foi o modelo cinético de pseudo-segunda ordem.

Tabela Erro! Use a guia Página Inicial para aplicar 0 ao texto que deverá aparecer aqui.1 - Parâmetros cinéticos para a adsorção do corante reativo Azul BF-5G.

\begin{tabular}{cccc}
\hline & \multicolumn{3}{c}{ Parâmetros } \\
\cline { 2 - 4 } Modelo & $\mathbf{K}^{*}$ & $\begin{array}{c}\mathbf{q} \text { eq } \\
(\mathbf{m g} / \mathbf{g})\end{array}$ & $\mathbf{R}^{\mathbf{2}}$ \\
\hline Pseudo 1 ${ }^{\mathrm{a}}$ Ordem & $2,09 \pm 0,02$ & $5,7 \pm 0,1$ & 0,9542 \\
Pseudo 2 ${ }^{\text {a Ordem }}$ & $0,040 \pm 0,005$ & $6,7 \pm 0,2$ & $\mathbf{0 , 9 7 2 6}$ \\
Intrapartícula & $2,09 \pm 0,08$ & $-0,16 \pm 0,01$ & 0,9768 \\
\hline
\end{tabular}

$\mathrm{K}^{*}=\mathrm{k}_{1}$ pseudo-primeira ordem $\left(\mathrm{h}^{-1}\right), \mathrm{k}_{2}$ pseudo-segunda ordem $\left(\mathrm{g} \cdot \mathrm{mg}^{-1} \cdot \mathrm{h}^{-1}\right)$ e $\mathrm{k}_{\mathrm{i}}$ difusão intrapartícula $\left(\mathrm{mg} \cdot \mathrm{g}^{-1} \cdot \mathrm{min}^{-0,5}\right)$ 
Segundo Ho e McKay (1999), quando o modelo de pseudo-segunda ordem representa a cinética, a adsorção química pode ser o mecanismo predominante no processo. De acordo com Ho et al. (1999), a razão desse comportamento pode ser atribuída a menor competição para os sítios de adsorção na superfície, com concentração mais baixa. Em concentrações mais elevadas, a competição para os sítios ativos da superfície será elevada, consequentemente, menores quantidades de adsorção serão obtidas.

\subsection{Isotermas de adsorção}

A Figura 2 mostra as isotermas de adsorção de corante Azul Reativo BF-5G, e carvão ativado de Babaçu, para as temperaturas de $20^{\circ} \mathrm{C}, 30^{\circ} \mathrm{C}$ e $40^{\circ} \mathrm{C}$, e as curvas obtidas pelos ajustes dos modelos de isotermas de Langmuir, Freundlich, Radkee Praunsnitz, Redlich Perterson, Tóth e Sips.

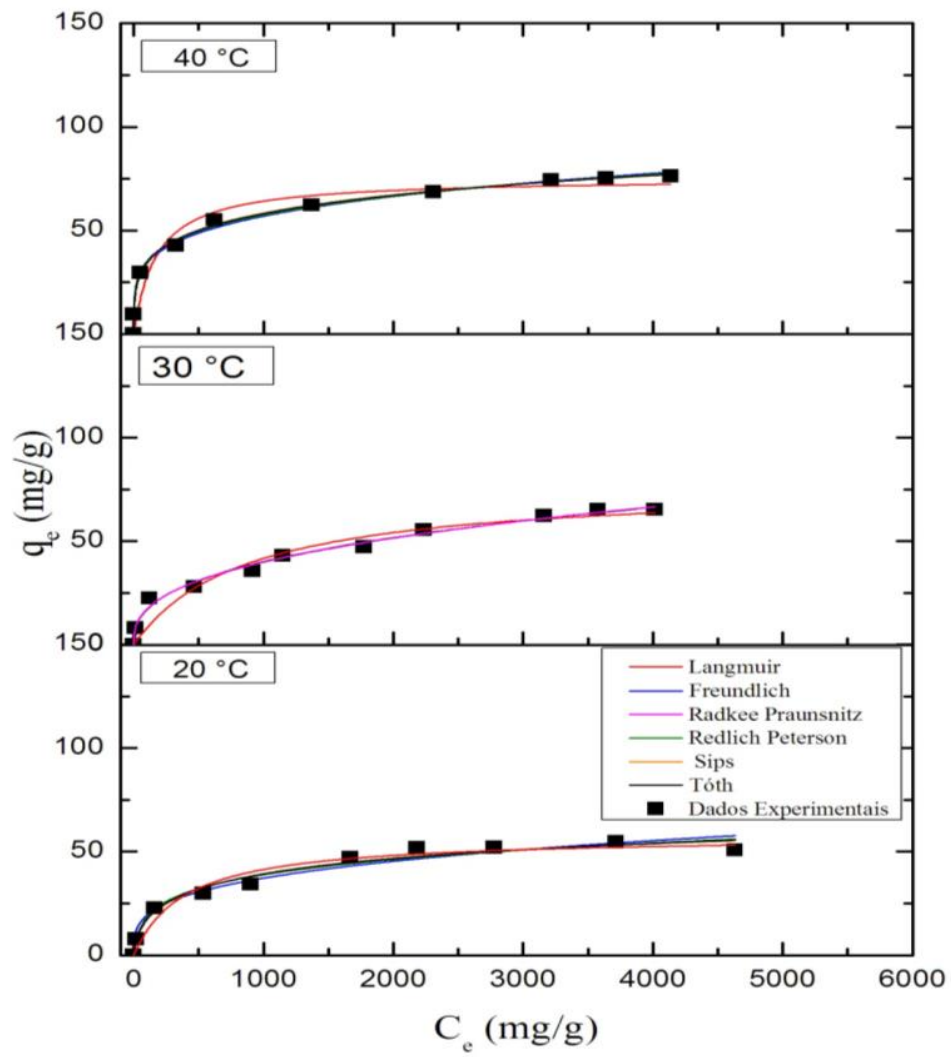

Figura 2 - Isotermas de Adsorção nas temperaturas de $40^{\circ} \mathrm{C}, 30^{\circ} \mathrm{C}$ e $20^{\circ} \mathrm{C}$.

Os valores dos parâmetros dos modelos de isotermas obtidos pelos ajustes aos dados experimentais de equilíbrio são apresentados na Tabela 2.

As isotermas apresentadas na Figura 2 mostram que a adsorção de corante Reativo Azul 
BF-5G em carvão ativado de Babaçu são do Tipo H2, para todas as temperaturas. Segundo Giles et al. (1960), esse tipo de isoterma é mostra que quanto mais sítios do adsorvente são preenchidos, torna-se cada vez mais difícil para a molécula de corante entrar em um sítio vazio disponível, ou seja, os sítios apresentam-se saturados entrando assim em equilíbrio.

As isotermas de adsorção para o carvão de Babaçu foram melhor ajustadas nos modelos de Freundlich em todas as temperaturas. As isotermas a $20{ }^{\circ} \mathrm{C}, 30{ }^{\circ} \mathrm{C}$ e $40{ }^{\circ} \mathrm{C}$ levaram os valores de $\mathrm{R}^{2}$ de 0,961; 0,9904 e 0,9950, respectivamente.

Sabe-se que a Isoterma de Freundlich é utilizada para sistemas heterogêneos com interação entre as moléculas adsorvidas. De acordo com o parâmetro n, conforme pode ser observado na Tabela 2 , que foi menor do que $1(0,22-0,37)$, há uma fraca interação entre o adsorvente e o adsorvato, acarretando baixas quantidades adsorvidas, indicando também ser um processo de quimissorção (VARGAS et al.,2011).

Tabela.2 - Valores dos parâmetros e correlações obtidos com o ajuste dos modelos de isotermas de adsorção em carvão ativado de Babaçu.

\begin{tabular}{|c|c|c|c|c|}
\hline \multirow{2}{*}{ Modelo } & \multirow{2}{*}{ Parâmetros } & \multicolumn{3}{|c|}{ Temperatura $\left({ }^{\circ} \mathrm{C}\right)$} \\
\hline & & 20 & 30 & 40 \\
\hline \multirow{3}{*}{ Langmuir } & $\mathbf{K}$ & $0,0025 \pm 0,0007$ & $0,012 \pm 0,0003$ & $0,006 \pm 0,002$ \\
\hline & $\mathbf{q}_{\text {máx }}$ & $58 \pm 3$ & $75 \pm 4$ & $77 \pm 7$ \\
\hline & $\mathbf{R}^{2}$ & 0,9565 & 0,9415 & 0,9454 \\
\hline \multirow{3}{*}{ Freundlich } & $\mathbf{K}$ & $5 \pm 1$ & $3,1 \pm 0,5$ & $12 \pm 1$ \\
\hline & $\mathbf{N}$ & $0,29 \pm 0,03$ & $0,37 \pm 0,02$ & $0,22 \pm 0,01$ \\
\hline & $\mathbf{R}^{2}$ & 0,9615 & 0,9904 & 0,9950 \\
\hline \multirow{4}{*}{$\begin{array}{c}\text { Radke e } \\
\text { Praunsnitz }\end{array}$} & $\mathbf{K}$ & $0,6 \pm 0,5$ & $*$ & $28 \pm 18$ \\
\hline & $\mathbf{F}_{\mathrm{rp}}$ & $10 \pm 5$ & $*$ & $13 \pm 1$ \\
\hline & $\mathbf{N}$ & $0,21 \pm 0,06$ & $*$ & $0,21 \pm 0,01$ \\
\hline & $\mathbf{R}^{2}$ & 0,9672 & $*$ & 0,9963 \\
\hline \multirow{4}{*}{$\begin{array}{l}\text { Redlich- } \\
\text { Peterson }\end{array}$} & $\mathbf{K}$ & $0,06 \pm 0,09$ & $*$ & $2 \pm 1$ \\
\hline & $\mathbf{q}_{\text {máx }}$ & $10 \pm 5$ & $*$ & $13 \pm 1$ \\
\hline & $\mathbf{N}$ & $0,79 \pm 0,07$ & $*$ & $0,79 \pm 0,01$ \\
\hline & $\mathbf{R}^{2}$ & 0,9673 & $*$ & 0,9963 \\
\hline \multirow{4}{*}{ Sips } & $\mathbf{K}$ & $0,02 \pm 0,01$ & $*$ & $0,06 \pm 0,01$ \\
\hline & $\mathbf{q}_{\text {máx }}$ & $79 \pm 22$ & $*$ & $176 \pm 48$ \\
\hline & $\mathbf{N}$ & $0,6 \pm 0,2$ & $*$ & $0,32 \pm 0,04$ \\
\hline & $\mathbf{R}^{2}$ & 0,9728 & $*$ & 0,9972 \\
\hline \multirow{4}{*}{ Tóth } & $\mathbf{K}$ & $0,3 \pm 0,4$ & $*$ & $2,0 \pm 0,5$ \\
\hline & $\mathbf{q}_{\text {máx }}$ & $103 \pm 62$ & $*$ & $765 \pm 845$ \\
\hline & $\mathbf{N}$ & $0,3 \pm 0,2$ & $*$ & $0,09 \pm 0,04$ \\
\hline & $\mathbf{R}^{2}$ & 0,9712 & $*$ & 0,9970 \\
\hline
\end{tabular}

\subsection{Estudo termodinâmico}

Os valores das propriedades termodinâmicas entalpia $\left(\Delta \mathrm{H}^{\circ}\right)$, entropia $\left(\Delta \mathrm{S}^{\circ}\right)$ e energia 
livre de Gibbs $\left(\Delta \mathrm{G}^{\circ}\right)$ foram calculados a partir das isotermas de adsorção de corante e são apresentadas na Tabela 3.

Observa-se por meio da tabela 3 que a energia livre de Gibbs a $40{ }^{\circ} \mathrm{C}$ apresenta um $\Delta \mathrm{G}^{\circ}$ negativo, característico de processo espontâneo, indicando que a temperatura favorece o processo de adsorção (WAN e ZHU, 2006) e mostrando a viabilidade do processo à medida que a temperatura aumenta (BACCAR et al., 2010).

Tabela 3- Propriedades termodinâmicas do processo de adsorção

\begin{tabular}{rcccc}
\hline Carvão & Propriedades & \multicolumn{3}{c}{ Temperatura $\left({ }^{\mathbf{0}} \mathbf{C}\right)$} \\
\cline { 3 - 5 } Ativado & Termodinâmicas & $\mathbf{2 0}$ & $\mathbf{3 0}$ & $\mathbf{4 0}$ \\
\hline \multirow{3}{*}{ Babaçú } & $\Delta \mathbf{G}^{\mathbf{0}}(\mathbf{k J} / \mathbf{m o l})$ & 2,48 & 1,82 & $-3,09$ \\
& $\Delta \mathbf{H}^{\mathbf{0}}(\mathbf{k J} / \mathbf{m o l})$ & & 83,4 & \\
& $\Delta \mathbf{S}^{\mathbf{0}}(\mathbf{J} / \mathbf{m o l} . \mathbf{K})$ & & 274 & \\
& & & & 274
\end{tabular}

Nota-se que o aumento da temperatura leva a um aumento da quantidade adsorvida no equilíbrio, característica de um processo endotérmico. Isto é comprovado pelos valores de $\Delta \mathrm{H}^{\circ}$. Processos endotérmicos têm como justificativa a formação do complexo ativado adsorvente/adsorvato na superfíce do carvão (HAMEED et al.,2007). Além disso, também é considerado um processo favorável em todas as temperaturas, como no caso do estudo de Cardoso et al. (2011), em que foi observado um aumento da quantidade adsorvida de corante remazol preto-B com o aumento da temperatura.

$\mathrm{O}$ valor positivo de $\Delta \mathrm{S}^{\circ}$ sugere um aumento da aleatoriedade na interface sólidosolução, originado de algumas mudanças estruturais em ambos, adsorvente e adsorvato, sugerindo afinidade dos adsorventes com os corantes (HILL, 1977).

\section{CONCLUSÕES}

No estudo da cinética de adsorção verificou-se que aproximadamente em 18 horas o sistema encontrava-se em equilíbrio. O melhor ajuste aos dados experimentais foi do modelo de pseudo-segunda ordem, indicando um processo controlado por quimissorção, que envolve forças de valência por meio do compartilhamento ou troca de elétrons entre adsorvente e adsorvato.

As isotermas de adsorção foram melhor ajustadas pelos modelos de Sips e Freundlich, caracterizando sistema heterogêneo. Observou-se, também, que o valor de $n$ era menor que zero, indicando processo quimissortivo. A quantidade máxima adsorvida foi de $76,21 \mathrm{mg} / \mathrm{g}$ a $40{ }^{\circ} \mathrm{C}$.

Os parâmetros termodinâmicos $\Delta \mathrm{H}^{\circ}, \Delta \mathrm{G}^{\circ}$ e $\Delta \mathrm{S}^{\circ}$ foram avaliados e mostraram que o processo é endotérmico e há um aumento da aleatoriedade na interface sólido-solução, conforme os valores de $\Delta \mathrm{H}^{\circ}$ e $\Delta \mathrm{S}^{\circ}$ obtidos. Pelos valores de $\Delta \mathrm{G}^{\circ}$ observou-se que o processo é espontâneo, mostrando que a temperatura favorece o processo de adsorção. 


\section{REFERÊNCIAS}

ALTINIS, I.K.; GUR ,A.; SEKI, Y.; "A natural sorbent, Luffa cylindrica for the removal of a model basic dye"; Journal of Hazardous Materials V.179, p. 658-664, 2010.

BACCAR, R.; BLÁNQUEZB, P.; BOUZIDA, J.; FEKIC ,M.; SARRÀ, M. "Equilibrium, thermodynamic and kinetic studies on adsorption of commercial dye by activated carbon derived from olive-waste cakes". Chemical Engineering Journal, v. 165, n. 2, p. 457-464, 2010.

BRÁS, R.; DIAS, J.; REGO, G.; et al., “Degradação de azo corantes por processo biológicos”. Revista Química Têxtil, V.68, p. 5-10, 2002.]

GILES, C. H.; MACE WAN, T. H.; NAKHWA, S. N.; SMITH, D.; J. Chem. Soc,3973, 1960.

HAMEED, B.H.; AHMAD, A.A.; "Batch adsorption of methylene blue from aqueous solution by garlic peel, an agricultural waste biomass", J. Hazard. Mater.V.164, p.870-875, 2009

HILL, C. G. "An introduction to chemical Engineering Kinetics and reactors Design". John Wiley e Sons, EUA, 1977.

HO Y.S., MCKAY G., "Pseudo-second order model for sorption processes", Process Biochem. V.34, p.451-465, 1999.

HO Y.S., MCKAY G., "Kinetic models for the sorption of dye from aqueous solution by wood", Trans IChemE, V.76, Part B,1998.

MOUSSAVI, G.; KHOSRAVI, R.; "The removal of cationic dyes from aqueous solutions by adsorption onto pistachio hull waste".Chemical Engineering Research and Design, V.89, p. 2182-2189, 2011.

RAJI, C.; ANIRUDHAN, T.S.; "Batch Cr(VI) removal by polyacrylamide-grafted sawdust: kinetics and thermodynamics". Pergamon, V. 32, p. 3772-3780, 1998.

SENTURK, H.B.; OZDES, D.; DURAN, C.; "Biosorption of Rhodamine 6G from aqueous solutions onto almond shell (Prunus dulcis) as a low cost biosorbent".Desalination, V.252, p. 81$87,2010$.

SUN, J.; SUN, S.; WANG, G.; QIAO, L.; "Degradation of azo dye Amido black 10B in aqueous solutions by Fenton oxidation process". Dyes and Pigments, V.74, p. 647-652, 2007.

VARGAS, A.M.M.;CAZETTA, A.L.; KUNITA, H.M.; SILVA, T.L.; ALMEIDA, V.C.; "Adsorption of methylene blue on activated carbon produced from flamboyant pods (Delonix regia): Study of adsorption isotherms and kinetic models",Chemical Engineering Journal ,V.168 ,p.722-730, 2011.

WANG S.; ZHU Z.H. "Effect of acidic treatment of active carbons on dye adsorption". Dyes and Pigments,p.1-9, 2006. 